\title{
Les enfants francophones, le langage et la communication
}

Alain Coianiz, Cécile Canut, Tayeb Bouguerra et Victor Allouche

\section{(2) OpenEdition}

Édition électronique

URL : http://journals.openedition.org/trema/1747

DOI : 10.4000/trema.1747

ISSN : 2107-0997

\section{Éditeur}

Faculté d'Éducation de l'université de Montpellier

\section{Édition imprimée}

Date de publication : 1 octobre 1999

Pagination : 115-126

ISSN : 1167-315X

\section{Référence électronique}

Alain Coianiz, Cécile Canut, Tayeb Bouguerra et Victor Allouche, «Les enfants francophones, le

langage et la communication », Tréma [En ligne], 15-16 | 1999, mis en ligne le 01 octobre 1999, consulté le 20 avril 2019. URL : http://journals.openedition.org/trema/1747 ; DOI : 10.4000/

trema. 1747

Ce document a été généré automatiquement le 20 avril 2019

Trema 


\title{
Les enfants francophones, le langage et la communication
}

\author{
Alain Coianiz, Cécile Canut, Tayeb Bouguerra et Victor Allouche
}

\section{Présentation du thème de l'atelier. Alain COÏANIZ}

1 Le vécu scolaire, le langage à l'école, sa pratique et la «conscience linguistique » à laquelle les enseignants se proposent de faire accéder les élèves, la communication scolaire marquée par ses codes et ses repères peuvent dessiner un espace nouveau, étranger ou inquiétant pour l'enfant d'une langue et d'une culture différentes. On peut tenter de tracer les contours de ce cheminement. Se posent alors des questions de fond: Comment « habiter » la langue? Que suppose communiquer dans cette langue ? Comment et pour quoi faire? De quelle socialisation (dans le milieu d'origine) vers quelle citoyenneté?

2 Si la citoyenneté suppose la socialisation, elle se donne à comprendre comme un déplacement des normes et références d'un individu du seul faire au croire, à l'adhésion morale, intellectuelle, psychologique du sujet à un idéal de valeurs organisatrices du groupe auquel il appartient. La question se pose avec acuité lorsque les «entités de participation sociale » (Goffman) relèvent de modèles sociaux diversifiés (les enfants du Mali, les Alukus et les Amérindiens de Guyane par exemple), sommés de s'inscrire à la fois dans des univers relationnels, axiologiques et symboliques bien souvent fort éloignés.

Pour ces enfants, étrangers d'Afrique et d'ailleurs, Français du lointain horizon que l'outre-mer circonscrit, par exemple, enfants socialisés, « citoyens » dans leurs groupes, les références et les valeurs officielles dont l'école est porteuse viennent interférer avec les leurs, si ce n'est les masquer, et parfois créer les conditions de leur destruction.

D'autre part, être citoyen, et de France métropolitaine, suppose un rapport dialectique à l'identité dont la notion même de «citoyenneté française» est le véhicule: quels personnages, quels objets symboliques d'investissement communs, quelles pratiques, quelle histoire communes peuvent constituer l'assise indispensable à ce qui se veut différent de la simple «nationalité »? Pour repenser la situation de la Guyane, plus 
particulièrement, qu'est-ce qui s'offre à l'élaboration symbolique commune, si ce n'est le kouac d'une part (la manioc) et la technologie d'autre part (les moteurs de pirogue) ? Et si l'école ne propose pas un langage d'élaboration d'une culture commune, par le jeu des mises en commun et des négociations des diverses représentations du monde, que peut-il advenir d'une citoyenneté affirmée formellement?

L'écart ainsi creusé, banal dans le rapport de forces assimilateur qu'il installe, se double d'un autre effet de reflet, celui que le vécu quotidien des valeurs de la pratique construit face à la citoyenneté officielle. C'est ainsi une double fracture que vit l'enfant français de l'ailleurs: celle de son adhésion aux structures de son groupe par rapport aux structures de la société française, et celle de l'ordinaire en face de la citoyenneté officielle.

Peut-on faire le pari qu'une des missions de l'école, au-delà de ses seules fonctions, est de construire une référence commune, verbalement élaborée, accédant ainsi à la conscience critique, par la mise en discours des différences et des ressemblances?

Un engagement de l'élève dans le travail scolaire, une participation aux activités ne peut se mettre en place qu'à certaines conditions, auxquelles sa culture d'origine le prédispose ou non:

- l'élève, dans une relation satisfaisante, doit disposer d'une capacité à se distancer de ses performances : c'est là la condition fondamentale du retour sur la production, de la mobilisation des acquis aux fins de vérification, d'amendement ;

- comme il structure son apprentissage dans une relation institutionnelle, donc sociale, il lui faut prendre en compte autrui, et la décentration empathique constitue une condition de base de son activité ;

- de plus, l'apprentissage se fondant sur une médiation, il évolue sur un axe allant de la recherche d'une aide à la sollicitation d'une caution de la part du détenteur de la Loi et du Savoir;

- enfin, l'adaptabilité, la curiosité, la possibilité de risquer l'image de soi et la place occupée, l'émergence d'un projet, son rapport à la subjectivité (sa disposition à opérer des choix personnels) et la plasticité de ses représentations (la possibilité de les travailler et de les faire évoluer) viennent compléter, selon nous, les conditions d'une communication didactique satisfaisante ;

- on n'omettra pas le fait que tout apprentissage réel bouleverse dans des mesures variables les codes acquis, est donc porteur de risques (mais aussi de bénéfices qu'il s'agit de mettre en évidence par un travail en commun) et que nul ne saurait s'y engager s'il n'est mû par un besoin ressenti de remédier à une tension, à un état de déséquilibre ressenti dans son rapport avec l'environnement, que seuls des acquis nouveaux pourront l'aider à surmonter. Montpellier III), aborde le thème suivant.

\section{Apprentissage et interculturalité : les enfants maliens face à l'école en français. Cécile CANUT}

9 Notre intervention propose quelques réflexions sur la relation du sujet bilingue/ plurilingue aux langues et sur l'apprentissage du français en situation multiculturelle africaine, et plus particulièrement malienne. La situation scolaire est très préoccupante dans ce pays puisqu'un enfant sur trois parvient à la sixième année, et seulement un sur dix à l'université, malgré les efforts financiers considérables de l'État malien (un tiers du 
budget est consacré à l'éducation). Signalons rapidement que l'enseignement est proposé en français, langue officielle parlée par à peine $10 \%$ des locuteurs et essentiellement en situation formelle, le bambara étant la langue véhiculaire majoritairement utilisée. Douze autres langues possèdent le statut de langues nationales. Seules quatre d'entre elles (bambara, songhay, peul et tamasheq) sont intégrées dans les écoles expérimentales bilingues.

L'hypothèse avancée est la suivante : le cadre socio-historique et linguistique du Mali similaire à de nombreux autres pays d'Afrique Noire - nécessite de considérer le rapport des enfants aux langues en présence comme spécifique eu égard à d'autres situations plurilingues, comme celles des pays maghrébins par exemple. Cette particularité, tant au niveau des représentations que des pratiques communicatives, peut bien sûr avoir des incidences sur l'apprentissage du français en France pour les jeunes maliens, surtout s'ils ont vécu dans leur pays d'origine avant leur arrivée.

11 Rappelons brièvement les points qui nous paraissent être symptomatiques de cette différenciation. Le statut des langues maliennes tout d'abord est révélateur : ces langues bien que "maliennes» et nationales ne correspondent pas à des «langues d'État» puisqu'elles sont très peu valorisées dans le domaine institutionnel et public, à l'inverse du français. L'absence de diffusion de l'écrit et des normes pour ces langues dont la normalisation/standardisation est très récente entraîne des perceptions sociolinguistiques très particulières : l'écrit est systématiquement assimilé au français, lui-même devenu synonyme d'école et d'ascension sociale. L'absence d'homogénéisation et de normes prescriptives pour les langues maliennes aboutit, à l'inverse, à des représentations divergentes : le rapport aux langues et les processus de symbolisation se construisent dans la pluralité, la variation et l'hétérogénéité. Ainsi, lors de sa rencontre avec le français à l'école (entre 4 et 6 ans), l'enfant doit inscrire son apprentissage dans un cadre homogène (une langue, langue de l'État, officielle), dans un rapport nouveau, celui de l'écriture (culturellement absent auparavant) et au sein d'une perspective homogénéisante à l'intérieur de cette langue (une norme, un « bon usage » qui s'oppose à l'appropriation du français des locuteurs de son pays, le français «du Mali»). Ces perceptions linguistiques antithétiques (très schématiquement : français $=$ écrit $=$ norme = école, langues maliennes $=$ oral $=$ variation $=$ communication $)$, dues à la récente standardisation des langues et à l'absence de politique linguistique forte en la matière (très peu de relais institutionnels pour la diffusion des langues maliennes: centres d'alphabétisation et écoles expérimentales), sont inscrites dans le cadre plus vaste de la divergence entre deux modèles, à la fois opposés et complémentaires, de la citoyenneté : occidental et africain (traditionnel). Les ajustements entre ces deux pôles sont en permanente évolution mais entrânent inévitablement des difficultés: le sujet, pris au piège de cette double contrainte (ascension sociale - français-modèle occidental / respect des traditions - langues maliennes-modèle africain), tente de le dépasser en «bricolant " un syncrétisme de rigueur. On le voit, la perception de l'écart sociopolitique très large entre langues maliennes et français (le phénomène est valable pour d'autres pays africains) accentuée par la vision idéologique occidentale effective depuis les colonisations (surévaluation des langues écrites valant comme seules "langues", péjoration des langues dites « orales », valorisation de la norme, système de valeurs établi sur les bases de l'homogénéisation, etc.), montre la singularité du rapport du sujet malien aux langues, qui n'est pas sans conséquence sur son apprentissage du français, perçu 
comme «utilitaire», et pour lequel il ne va pas nécessairement mobiliser un profond désir d'appropriation.

\section{La prise en charge de la citoyenneté par l'école algérienne. Tayeb BOUGUERRA}

Si la langue arabe ne connaît pas le terme citoyen qu'elle traduit par muwatan signifiant compatriote, les textes fondateurs d'expression française (Charte nationale, Constitutions...) font un usage récurrent du terme citoyen (et plus rarement de citoyenneté). En discours politique, le terme citoyen est généralement suivi de l'expansion adjectivale socialiste.

14 L'acception de citoyen et de citoyenneté focalise moins sur la définition juridique ou statutaire (droits sociaux et devoirs), effective (qui réfère à la mise en œuvre des principes de citoyenneté : prise en charge, couverture médicale, retraite, etc.) que sur une acception d'ordre politique : le citoyen à former est un citoyen musulman, nationaliste, socialiste engagé dans la lutte anti-impérialiste et dans l'œuvre d'édification socialiste. C'est dire si la mission cardinale de l'école algérienne est double : dispenser un savoir général (maîtrise des savoirs fondamentaux) et former le futur citoyen, conformément aux valeurs arabo-islamiques (au passage la référence à la dimension berbère est gommée) et aux valeurs de révolution de 1954.

Dans ce contexte éminemment politique, la langue arabe représente un enjeu considérable. L'enseignement de l'arabe, langue nationale et officielle, langue du sacré, participe des mêmes visées idéologiques : unification de la nation et formation du citoyen musulman - des cours d'éducation islamique sont dispensés dès la quatrième année du primaire; dans les lycées des séries Sciences islamiques voient le jour, de nouvelles universités ont pour mission d'assurer la formation des futurs Imams (théologiens). L'arabisation, considérée comme récupération de la langue nationale, procède de la volonté de parachever l'indépendance politique par l'indépendance linguistique. L'arabisation progressive de l'environnement, du système éducatif, juridique, administratif, représenteront les principales étapes de cette vaste entreprise. Cette volonté d'unification se traduit par un centralisme (examens et diplômes nationaux), uniformisation des démarches jusque dans les modes d'exploitation didactique.

Quant aux objectifs assignés à l'enseignement des langues étrangères, ils sont essentiellement d'ordre pragmatique (favoriser le dialogue nord-sud, le transfert des technologies, "permettre l'accès au savoir scientifique et technique ») ou civilisationnel (" ouvrir sur le monde», "dialogue des cultures »...). Au niveau pédagogique, cette volonté politique est manifeste d'emblée dans les manuels. Les supports choisis présentent des modèles de bons citoyens : paysans citoyens (se dévouant à accroître la production), militaires citoyens (veillant jalousement sur les frontières), femmes citoyennes (mobilisées et entreprenantes), cadres citoyens (dynamiques, conscients et responsables), médecins citoyens (se sacrifiant pour soigner les malades), enseignants citoyens (se battant contre l'ignorance...) l'ensemble des personnages modèles exerce une citoyenneté tissée de responsabilité, d'engagement militant, de respect d'autrui, de solidarité, d'entraide. 
17 Cette même volonté politique rend compte de l'évolution du statut du français et des différentes ruptures qui se sont opérées dans le champ de son enseignement. La hantise de l'aliénation a conduit les décideurs à privilégier dans les années 1970 les auteurs engagés (Zola, Germinal ;Vallès, L'Insurgé), qui forment à une parole revendicatrice, à rejeter dans les années 1975 le littéraire parce que connoté idéologiquement (Flaubert, L'éducation sentimentale ; Stendhal, Le Rouge et le Noir ;Molière, Dom Juan),à favoriser en 1976 l'avènement du français fonctionnel qui fit entrer en classe de nouveaux supports didactiques (moulin à café, carburateur, pneu, sèche-cheveux, etc.)

Dans le système éducatif algérien, l'acception de citoyenneté focalise essentiellement la dimension politique : le bon citoyen n'est pas forcément celui qui paie ses impôts, qui effectue son service militaire, mais surtout celui qui adhère pleinement aux valeurs prônées par le système : nationalisme, socialisme, engagement anti-impérialiste. En ce sens, la citoyenneté se confond avec le militantisme. On notera que les valeurs telles que la tolérance, la laïcité, les libertés syndicales, le droit de grève, le droit de manifester n'entrent pas dans l'acception de la citoyenneté. La dimension participative de la citoyenneté se limite à l'expression de la solidarité, à l'engagement et à la lutte contre l'analphabétisme, à la participation aux grandes entreprises d'édification, participation aux campagne d'explication (construction du barrage vert, lutte contre la désertification, participation aux campagnes d'explication de la révolution agraire...). D'une manière générale, le discours didactique n'entraîne pas à une prise de parole citoyenne, à une parole revendicatrice, tant l'objectif est de développer une parole communautaire, la parole du parti unique.

19 Aujourd'hui, avec l'avènement de la démocratie et de ses différentes manifestations que sont le multipartisme, la presse privée, la libre circulation des individus (suppression de l'autorisation de sortie du territoire), on ose espérer que le système éducatif prendra en charge l'enseignement d'une véritable citoyenneté fondée sur l'adhésion aux valeurs fondamentales, sur l'apprentissage d'une citoyenneté participante.

20 (Alain Coïaniz) La question qui, insistante, vient se poser aux didacticiens, dans cette problématique, est, au fond, celle des rapports au langage et Victor ALLOUCHE (Université Paul Valéry) propose ses réflexions sur le thème :

\section{Habiter la langue. Victor ALLOUCHE}

21 À partir de quel moment peut-on dire qu'un apprenant habite la langue étrangère qu'il tente d'acquérir?

À partir du moment où il a incorporé les signifiants pour entrer dans les représentations, et à partir du moment où les mots sont entrés dans la vie affective. Cette entrée peut se décrire linguistique ment et psychologiquement.

\section{1) Linguistiquement}

La configuration du signe saussurien ne permet pas de rendre compte de son incorporation. Saussure prend un point de vue d'extériorité par rapport à la langue, fait du rapport signifiant/signifié un rapport arbitraire. Dans notre conception, on pourrait parler d'arbitrarité pour une langue étrangère pas encore incorporée, non une langue maternelle. 
Or que se passe-t-il quand on apprend une langue étrangère?

On peut observer trois configurations du signe qui représentent des niveaux de plus en plus importants d'incorporation de la langue.

La première configuration est caractérisée par une approche du signifiant linguistique, vocal, phonologique, scriptural. Il s'agit là d'une tentative qui ramène toujours au corps de l'autre, le parleur en LE, dans la mesure où on n'accède à aucune représentation.

La seconde configuration est caractérisée par la séparation plus marquée que chez Saussure du signifiant et du signifié. Nous séparerons les deux termes de trois ou quatre barres pour la symboliser. La liaison de la forme et du sens n'est pas encore faite. Le rapport est distendu. La mémoire fait son travail difficile. Les habitudes sont longues à acquérir.

La troisième configuration est caractérisée par l'incorporation complète du signifiant et l'entrée de l'apprenant dans la représentation/ signifié. C'est l'inversion du rapport saussurien ou ce que Bakhtine appelle le processus de compréhension. Le signal bakhtinien est enfoui sous le signe, autrement dit le signifiant est enfoui sous le signifié. $C^{\prime}$ est cette troisième configuration qui correspond à une langue habitée, à une incorporation du signe, et nous placerons alors le signifié au-dessus du signifiant.

\section{2) Psychologiquement}

La symbolisation linguistique d'une langue étrangère signale que l'apprenant est entré dans la vie affective en langue étrangère. Qu'est-ce que cela signifie ?

Cela signifie que l'incorporation de la langue étrangère ne peut pas être séparée de l'affectivité ressentie pour la langue et les êtres qui la parlent.

Je parle d'affectivité parce que tous les mots ont des auteurs et que l'emploi des mots signifie la relation et l'acceptation des auteurs étrangers. On apprend les mots dans les relations sociales et donc dans une relation à autrui. C'est autrui qui nous permet d'habiter les mots ou de ne pas les habiter parce que nous éprouvons des sentiments de rejet ou au contraire de sympathie.

"Customer" a d'abord rencontré " coutume » en classe de langue avant d'être naturalisé par un serveur derrière son comptoir, à qui je parlais de /klaijnt/, "client » avec une prononciation anglicisée. «I see you » a rencontré Emma, petite fille blonde aux yeux rieurs et aux joues roses, au jeu de cache-cache.

bolisation linguistique serait un autre nom de la production des signes et une entrée dans la signifiance parce que fabriquer du sens en LE, c'est d'une certaine manière accepter de se faire représenter par cette LE et de partager les mots d'une communauté d'auteurs, auteurs qui m'ont permis d'apprendre les mots.

Il faut ajouter à cela que ces relations affectives sont au cœur du processus psychologique dans la mesure où il s'agit pour un apprenant donné de remplacer sa langue maternelle, même momentanément, nouée à lui de façon affective. D'où l'idée que le deuxième aspect symbolique relève de l'affectivité pour les mots eux-mêmes puisqu'il y a une acceptation ou non - de remplacer les mots de la LM, autrement dit les mots de la LM condensent de l'affectivité: «J'ai eu beaucoup de plaisir à utiliser le mot 'slippery' pour remplacer le mot

Tréma, 15-16 | 1999 
〈glissant` français, parce que j'ai eu beaucoup de mal à utiliser le mot 'roll' pour remplacer le mot 〈petit pain français. »

Ainsi, habiter la langue présuppose que ce travail affectif a pu avoir lieu.

(Alain Coïaniz) Parler d'affectivité, on l'a vu, implique le corps.

Jacques GLEYSE de l'IUFM de Montpellier, s'interroge sur les rapports entre :

\section{Corps, savoir, langage et citoyenneté. Jacques GLEYSE}

L'intervention est articulée autour de cinq questions fondamentales :

- Le corps possède-t-il un langage?

- Le corps est-il signifiant?

- Le corps est-il porteur d'un savoir?

- Le corps dit-il la citoyenneté ?

- Y a-t-il une régularisation et une police des corps dans la société et dans l'école?

Chaque question appelle une réponse nuancée et des questions subsidiaires.

1) Ainsi pour le premier point il est possible de poser quatre questions complémentaires et d'apporter de rapides réponses.

La première est : $\mathbf{y}$ a-t-il un langage du corps ?

La réponse est relativement simple à apporter. Non, il n'y a pas de langage du corps au sens habituellement attribué à ce terme. Le langage du corps n'est pas un support d'interactions "universalisables ", sauf dans le cas où il a été explicitement construit comme tel (le langage des sourds, utilise le support de la lumière au lieu du son).

La deuxième peut être ainsi formulée : le corps participe-t-il au langage verbal ? Oui, bien sûr ; tous les travaux de " praxématique » et de « biolinguistique » le montrent.

5 La troisième sous-question est la suivante : Le langage est-il issu de transformations et de techniques corporelles? Depuis L'homme et la matièreet surtout Le geste et la parole d'André Le-roy-Gourhan, cela ne fait plus aucun doute. C'est parce que la main libère la mâchoire de la tâche de tuer et de déchirer que la structure corporelle (le pharynx) peut progressivement se modifier et produire un langage au sens où nous l'entendons.

Enfin, l'on doit se demander si le langage et l'action corporelle sont liées? Dans la perspective " énactive » de Varella, on doit très probablement considérer qu'action et pensée sont largement indissociables (même si la deuxième peut être l'inhibition de la première) et en ce sens par extrapolation, acte et langage ne pourraient être radicalement séparés.

2) La deuxième grande question implique au moins deux réponses.

Le corps est, comme l'écrit Jean-Marie Brohm, «le signifiant flottant par excellence »ou, pour le dire comme Michel Bernard, «le symbole dont use une société pour parler de ses fantasmes». Le corps porte donc toutes les marques, tous les savoirs aussi d'une société, d'un groupe social, d'un groupe particulier, d'une famille... Il dit la ruralité, l'urbanité, la classe sociale, l'éducation, le niveau de culture, le genre. Il témoigne ou non de pratiques corporelles marquées elles aussi par des déterminants sociaux ( $C$ f., P. Bourdieu, La distinction).

Mais, le corps dit peut-être aussi, selon notamment les théories de Georg Groddeck (Cf., Le livre du ça ),ce que le langage verbal ne dit pas. Les douleurs, ulcères, lombalgies, 
rhumatismes, voire les rhumes, grippes (Laborit va même jusqu'aux cancers), mais aussi certains accidents (considérés alors comme des lapsus) expriment ce que le langage n'a pu dire.

3) La troisième question ne peut que trouver une réponse positive dans la perspective de la citoyenneté.

Oui, le corps est porteur d'un savoir. Avec Marcel Mauss (Sociologie et anthropologie)on sait que les techniques du corps sont inscrites dès le plus jeune âge dans la structure corporelle. Il existe différentes façons de se nourrir, se vêtir, dormir, se coiffer, faire l'amour, saluer, se situer, regarder, selon les groupes culturels (au sens anthropologique). Les techniques sont des savoir-faire traditionnels efficaces qui permettent d'être reconnus comme appartenant à une culture. Mais on peut aller plus loin encore et évoquer avec Norbert Elias (La civilisation des mours)la perspective d'une civilité profondément incorporée, en Occident (mais aussi ailleurs, elle n'est pas tout à fait identique). Le rapport aux contacts (le contact autorisé avec seulement certaines parties du corps, l'usage de la fourchette ou même de la main droite), à la «monstration » des parties du corps, aux fèces, aux excrétions, aux flatulences, aux éructations, à la miction, à la nourriture est régi par un savoir précis qui s'est bien souvent " psychologisé » par le biais du système émotionnel. Des manifestations impromptues ou non conformes aux normes dans les domaines évoqués plus haut provoquent honte, malaise...

Ce savoir, même s'il peut être discuté (et s'il peut être différent dans d'autres lieux du monde), s'apprend dès le plus jeune âge.

4) A l'avant dernière question : "le corps peut-il dire la citoyenneté ?", il semble possible d'apporter un réponse plutôt positive.

Si l'on admet que la figure du citoyen a été jusqu'aux dernière décennies le «bourgeoismâle-propriétaire-adulte ». Ce prototype suppose une certaine incorporation de normes, une certaine forme de "carcéralisation " de la corporalité. Et même si la citoyenneté aujourd'hui se décline selon d'autres logiques (femmes, enfants, globalisation...), l'habitus corporel valorisé comme citoyenneté, n'est sans aucun doute pas neutre. On doit de nouveau renvoyer la réflexion vers Norbert Elias et les normes de civilité (en Chine l'on crache au réveil, pas en Occident, en Orient on rote après un bon repas, pas en Occident du moins le cache-t-on. Il faut aussi évoquer le rapport à la violence, différent selon les groupes). Bien sûr, ces normes peuvent être rediscutées et peuvent être redistribuées mais l'on ne peut refuser a priori l'idée qu'elles participent à une position citoyenne.

41 Il en va de même de l'habitus.Selon P. Bourdieu, il est aisé de repérer «l'aristocrate », le « bourgeois » ou le « prolétaire » par son habitusde classe. Et si la citoyenneté correspond à un habitusparticulier, l'écart à la norme dira ou non la citoyenneté.

Enfin, on ne peut pas passer sous silence la notion de «proxémique » élaborée par Edgar T. Hall dans La dimension cachée. A chaque situation et à chaque groupe sont associées des distances corporelles relativement précises (intimes, personnelles, sociales...). En Occident ces distances sont constantes et très respectées. Le comportement citoyen (même si l'on ne pense pas dans la citoyenneté le seul assujettissement, mais aussi l'aspect émancipatoire) implique de maîtriser avec précision et dans toute circonstance ces distances. 
Dans cette école adaptée d'Antécume Pata, en Guyane française, les jeunes wayanas apprennent à lire et à écrire dans leur langue maternelle, avant de poursuivre leur scolarité en français.

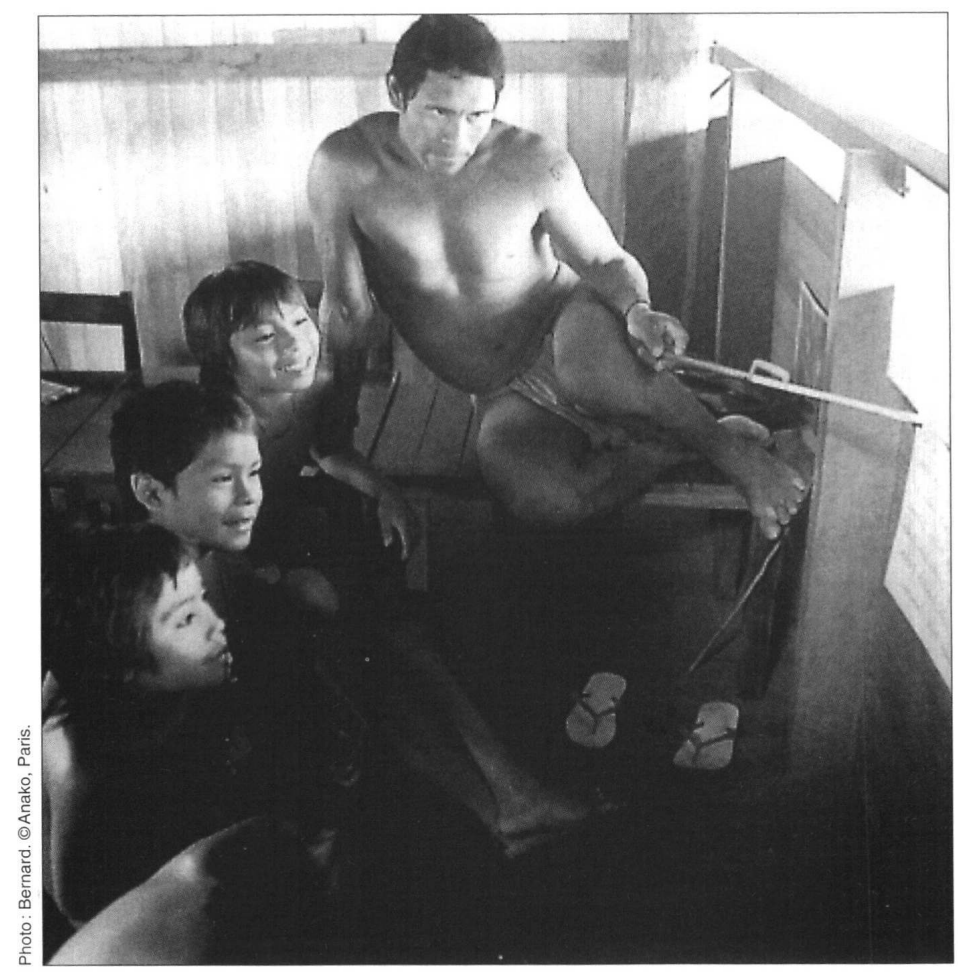

Le Courrier de I'UNESCO. Paris, UNESCO, avril 1995 (Vol. 48, №4), 50 p., p. 29

5) A la dernière question la réponse ne peut-être que positive. Il n'est pas possible de minimiser les micro-pouvoirs qui s'exercent sur l'ordre du corps et qui peuvent revenir bien souvent à la colonisation d'un groupe dominé par un autre détenteur du pouvoir, de l'argent ou du langage.

Ce système de régularisation et de police des corps est mis en place à l'école sous la forme de la norme du "silence et de l'immobilité ", si bien décrite par Daniel Denis (TRÉMA, spécial Hors-série Comment peut-on enseigner une culture corporelle ?). Dans la société, il prend la forme de régularisation des formes autant que des comportements. Pour les filles, un récent travail de recherche, montre que la forme de «la poupée Barbie performante » est un modèle identificatoire quasiment totalitaire dès la crise pubertaire. Pour les garçons le modèle serait plutôt « le sportif qui ne tombe jamais » (ou qui ne perd jamais). Les procédures pour parvenir à ces formes idéalisées sont multiples : pratiques corporelles bien sûr, mais aussi chirurgie esthétique, régimes amincissants, orthopédie et orthodontie... En général, il s'agit de faire souffrir la chair, le corps, comme l'ont fait précédemment la plupart des religions (flagellations...) et d'éveiller ainsi le sentiment du péché corporel.

Il serait évidemment possible de développer encore ce point en mettant en évidence une police des mœurs, mais aussi de la bouche (sourire, ne pas crier...). Tous ces aspects contribuent à la mise en place d'une régularisation du corps, d'un assujettissement de celui-ci évacuant largement la perspective émancipatoire du positionnement citoyen. 


\section{Débat et conclusions}

Face aux dimensions qui composent la notion de socialisation, on peut se demander dans un premier temps si la citoyenneté n'est pas à saisir fonctionnellement comme une production sociale de stabilisation, une forme en quelque sorte jugulatoire des risques de débordement des subjectivités.

En effet, la citoyenneté s'accommode d'arrangements avec le ciel (changeant) des politiques, et l'on peut distinguer des niveaux de citoyenneté : alors que la Guyane est reconnue comme département en 1947, la plus grande partie de son territoire (le territoire intérieur de l'Ininni) conserve un statut particulier, avec un gouverneur, à l'instar des colonies... Que dire de la reconnaissance «en bloc » de certaines de ses populations comme citoyens français (alors que traditionnellement ce statut fait l'objet d'une demande individuelle), ou encore des "tolérances» dont bénéficient certaines communautés, amérindienne ou boni, concernant l'école ou le service militaire? Pourquoi, aujourd'hui dans les écoles primaires, sous l'égide du Recteur, des médiateurs dont certains ne possèdent pas le baccalauréat? Pourquoi un salaire minimum au montant inférieur à celui de la métropole?

4 À côté des pratiques de socialisation, dont celle de l'école, œuvrant à la construction d'une culture commune et à sa verbalisation, il semble que le discours de la citoyenneté se constitue en discours officiel, idéalisé, conscient et proposé aux projets individuels, construit même en objet de désir, à revendiquer, pour ceux qui n'en bénéficient pas. Mais, à vrai dire, le « ressortissant » français n'est-il pas, de fait, citoyen français ? Il semblerait qu'aux modèles sociaux de l'ordinaire se surajoutent des modèles d'excellence susceptibles d'être marqués au coin de l'estampille - du label - de citoyenneté.

Citoyen, l'homme social est sujet d'une communauté de parole, d'une communauté discursive, capable de décentration, de distanciation, de critique argumentée, et capable de construire - aussi - linguistiquement son espace d'autonomie relative, de sujet social. Il n'est de citoyenneté, dans le cadre qui nous occupe - celui de la langue, de son enseignement et de son apprentissage - que dans la légitime distance, conscience et maîtrise des formes d'expression d'une subjectivité qui non seulement s'intègre aux lois, mais encore prend part à leur élaboration, leurs limites, leur progrès.

\section{RÉSUMÉS}

Le vécu scolaire, le langage à l'école, sa pratique et la "conscience linguistique » à laquelle les enseignants se proposent de faire accéder les élèves peuvent dessiner un espace nouveau. Etranger ou inquiétant pour l'enfant d'une culture différente. Sont évoqués ici les contours de ce cheminement.

The school experience, language at school, its actual use as well as the "language awareness" towards which end teachers offer to help students reach may represent a new conceptual sphere 
which the child being from a different culture may find strange or threatening. This paper evokes the outlines of this intellectual journey.

INDEX

Keywords : interculturality, schooling

Mots-clés : interculturalité, scolarisation

\section{AUTEURS}

ALAIN COIANIZ

Professeur, Université Montpellier III

\section{CÉCILE CANUT}

Université Montpellier III

TAYEB BOUGUERRA

Université Montpellier III

VICTOR ALLOUCHE

Université Montpellier III 